\title{
Do a Neurocritical Care Unit Requires Dedicated Nurse Staff?
}

\author{
Georgia Tsaousi ${ }^{1^{*}}$ and Federico Bilotta ${ }^{2}$
}

${ }^{1}$ Department of Anesthesiology and ICU, Faculty of Medicine, Aristotle University of Thessaloniki, Greece

2Department of Anesthesiology, University of Rome "La Sapienza", Rome, Italy

"Corresponding author: Georgia Tsaousi, Anesthetist-Intensivist, Assistant Professor of Anesthesiology, Faculty of Medicine, Aristotle University of Thessaloniki, , Maiandrou 32, GR 56224 Thessaloniki Greece, Tel. +30 2310 994855, Fax+30 2310994860, E-mail: tsaousig@otenet.gr

Rec date: Oct 29, 2015; Acc date: Nov 4, 2015; Pub date: Nov 12, 2015

Copyright: (C) 2015 Tsaousi G, et al. This is an open-access article distributed under the terms of the Creative Commons Attribution License, which permits unrestricted use, distribution, and reproduction in any medium, provided the original author and source are credited.

\section{Introduction}

Neurocritical care (NCC) has emerged as a distinct medical specialty that links neurology, neurosurgery, and critical care medicine in the comprehensive management of complex and life-threatening neurological problems [1]. As the field has evolved from a primary focus on the postoperative care of elective neurosurgical patients, nowadays its primary focus has moved to the resuscitation of neurological emergency cases like traumatic brain injury (TBI) or subarachnoid hemorrhage (SAH) that still remain a clinical challenge [2].

Albeit primary brain insult constitutes a crucial component of patient's total physical and cognitive deterioration found in neurological emergencies, prevention of secondary brain injury induced by altered cerebral hemodynamic and leading to a mismatch between cerebral demand and supply is of paramount importance $[2,3]$. In most of the cases, secondary injury management is more daunting task and incurs a higher incidence of morbidity and mortality than the primary injury itself.

In order to deal with the needs of the high acuity population in contemporary NCC setting, a multidisciplinary team management is considered of paramount importance; as members of this team nurses hold a key-role for patient care optimization. Albeit, all members of the neuro-oriented team (intensivists, anesthesiologists, neurologists, nurses) assess patients' clinical status throughout the day, nurses as the bedside caregivers are best suited to more frequent checks of patient's clinical status.

Ideally, a neuroscience ICU nurse should be qualified with an increased knowledge base and possess both technical and interpersonal skills to ensure safe care. Nursing care priorities should focus on initial and ongoing assessment, monitoring, hemodynamic management and stabilization of the patient admitted in the NCC. Provision of regular neurological exams, vigilance and diagnosis of postoperative delirium, [4] interpretation of vital signs and multimodal brain monitoring indications, [5] correct placement of height of drain, lowering abrupt intracranial pressure (ICP) increases, checking the ventilation trend, and safely transporting the patient to either CT / MRI / angiography suite or operating theater are many of the multilevel practices NCC nurses are challenged with on a daily basis. Perhaps the most important aspect of nursing care is to recognize the subtle changes that could potentially either induce or be indicative of derangement of cerebral hemodynamics, so appropriate interventions can be applied accordingly. This implies that NCC nurses should be aware of the importance of specific physiological parameters alterations in neuroscience critical care management. For instance, even mild systemic hypotension in specific neuro-populations such as
SAH and TBI might be more relevant than in other intensive care unit (ICU) patients, [6] while changes of arterial carbon dioxide promote alterations of cerebral blood flow [7]. Moreover, glucose levels should be aggressively treated, as there is evidence that both hypoglycemia and hyperglycemia have an adverse impact on neurologic outcome $[8,9]$.

The likelihood for alterations of patient's vital signs during daily nursing care is extremely high. Nursing tasks such as oral care, endotracheal suctioning, repositioning and chest physiotherapy are routinely performed in critically ill patients. All of these tasks are common and left to the discretion of the nurse as to when to perform, how, and for what duration. Though studies are limited, nurses might deliberately limit such tasks in patients with a concern for deleterious ICP increase. However, knowledge of practices for safe suctioning (such as endotracheal lidocaine instillation, one or two quick passes of the catheter, etc.) [10], application of adequate sedation and pain relief before providing patient care and chest physiotherapy, careful repositioning, controlling fever, seizures, prophylaxis of deep vein thrombosis are of paramount importance to anticipate or prevent common ICU complications and to avoid potential ICP elevation [11].

Beyond the care that aims to minimize neurologic status deterioration, nurses are delegated to check repeatedly throughout the day vital signs, intake and output (increased diuresis in case of central diabetes insipidus due to severe brain damage), all continuous infusions, patency of intravenous lines, depth of endotracheal tube results waveforms of all invasive monitors, lab work results (with special attention to electrolytes and glucose levels) and to take care of toileting and early mobilization. Furthermore, nurses should play a pivotal role in supporting family members and alleviate much of their anxiety.

In everyday practice, much of the decision making about the timing and the type of intervention needed to accomplish the predetermined goals is based on the judgment and experience of the bedside nurse with limited oversight from the attending physician. In case of a medical crisis, the bedside nurse is assigned with the duty to alert the responsible physician, for applying the indicated treatment modalities. Protocols implementation is particularly helpful in guiding less experienced ICU nurses and standardizing nursing practice, towards optimization of the quality of care.

Current literature research on outcomes of NCC patients suggests that the high-quality NCC management and the establishment of evidence-based protocols specifically oriented to treat complications and lessen secondary brain injury can affect favorably not only survival but also the quality of life after serious brain damage [3]. In conclusion, it becomes evident that a nurse staff dedicated and specifically trained 
Citation: Tsaousi G, Bilotta F (2016) Do a Neurocritical Care Unit Requires Dedicated Nurse Staff?. J Nurs Care 5: e128. doi: $10.4172 / 2167-1168.1000 \mathrm{e} 128$

Page 2 of 2

in the treatment of NCC patients, along with a subspecialty physician staff, can significantly improve the quality of clinical care in neuroscience critical care population, thus the need for such an expertise personnel is an absolute priority.

\section{References}

1. Rincon F, Mayer SA. (2007) Neurocritical care: a distinct discipline? Curr Opin Crit Care 13: 115-121.

2. Siegemund M, Steiner LA. (2015) Postoperative care of the neurosurgical patient. Curr Opin Anaesthesiol 28: 487-493.

3. Kurtz P, Fitts V, Sumer Z, Jalon H, Cooke J, et al. (2011) How does care differ for neurological patients admitted to a neurocritical care unit versus a general ICU? Neurocrit Care 15: 477-480.

4. Bilotta F, Lauretta MP, Borozdina A, Mizikov VM, Rosa G. (2013) Postoperative delirium: risk factors, diagnosis and perioperative care. Minerva Anestesiol 79: 1066-1076.

5. Le Roux P, Menon DK, Citerio G, et al. (2014) Consensus summary statement of the International Multidisciplinary Consensus Conference on Multimodality Monitoring in Neurocritical Care: a statement for healthcare professionals from the Neurocritical Care Society and the European Society of Intensive Care Medicine. Intensive Care Med 40: 1189-1209

6. Basali A, Mascha EJ, Kalfas I, et al. (2000) Relation between perioperative hypertension and intracranial hemorrhage after craniotomy. Anesthesiology 93: 48-54

7. Fox J, Gelb AW, Enns J, Murkin JM, Farrar JK, et al. (1992) The responsiveness of cerebral blood flow to changes in arterial carbon dioxide is maintained during propofol-nitrous oxide anesthesia in humans. Anesthesiology 77: 453-456.

8. Bilotta F, Rosa G. (2010) Glucose management in the neurosurgical patient: are we yet any closer? Curr Opin Anaesthesiol 23: 539-543.

9. Bilotta F, Rosa G. (2012) Glycemia management in critical care patients. World J Diabetes 3: 130-134.

10. Bilotta F, Branca G, Lam A, Cuzzone V, Doronzio A, et al. (2008) Endotracheal lidocaine in preventing endotracheal suctioning-induced changes in cerebral hemodynamics in patients with severe head trauma. Neurocrit Care 8: 241-246.

11. Tume LN, Baines PB, Lisboa PJ. (2011) The effect of nursing interventions on the intracranial pressure in paediatric traumatic brain injury. Nurs Crit Care 16: 77-84. 